\title{
Corneal integrity and thickness of central fovea after phacoemulsification surgery in diabetic and nondiabetic cataract patients
}

\author{
Zhuo Chen, Fanqian Song, Liyao Sun, Chuchu Zhao, Ningning Gao, Ping Liu, Hongyan Ge
}

Department of Eye, First Affiliated Hospital, Harbin Medical University, Harbin, China

Submitted: 5 January 2016

Accepted: 20 July 2016

Arch Med Sci 2018; 14, 4: 818-825

DOI: https://doi.org/10.5114/aoms.2016.64036

Copyright @ 2016 Termedia \& Banach

\section{Abstract}

Introduction: Here we intended to investigate the changes in corneal endothelial cells and foveal thickness after phacoemulsification surgery on the eyes of diabetic and non-diabetic cataract patients.

Material and methods: A total of 120 cataract patients who were scheduled for phacoemulsification surgery and intraocular lens implantation were recruited and divided into 2 categories according to the diagnosis of diabetes mellitus. Changes in integrity, endothelial cell density (ECD), coefficient of variation (CV), percentage of hexagonal cells (PHC), central corneal thickness (CCT), and central foveal thickness (CFT) were all recorded at preoperative day 1 and postoperative day 3, 1 week, 1 month, 3 months and 6 months. Results: None of the recorded variables showed any difference between the nondiabetic and diabetic groups before surgery $(p>0.05)$. During the postoperative 6 months, ECD and PHC decreased and CV increased in both groups (all $p_{\text {time }}<0.05$ ), whereas CCT and CFT fluctuated in both groups significantly (both $p_{\text {time }}<0.05$ ), with their individual peaks at postoperative 1 week in the diabetic group. The groups differed significantly in ECD, PHC, and CV at each time point postoperatively (all $p_{\text {group }}<0.05$ ). Furthermore, the diabetic group had improved CFT during the postoperative 1 month and higher CCT during the 6 months postoperatively than the nondiabetic group (all $p_{\text {group }}<0.05$ ). The time and group interactions were significant for ECD, CV, PHC, CCT and CFT (all $p_{\text {group } x \text { time }}<0.05$ ).

Conclusions: The diabetic group had more changes in corneal endothelial cells and foveal thickness than the nondiabetic group postoperatively.

Key words: phacoemulsification, corneal integrity, diabetes mellitus, endothelial cell density.

\section{Introduction}

Cataract is the most common cause of visual impairment in older adults aged 50 years or above, accounting for $33 \%$ of visual impairment and $50 \%$ of blindness globally [1]. Cataract surgery is one of the most common procedures performed worldwide to restore vision and has a high rate of success [2]. However, some surgery-related complications may occur during the early postoperative period after cataract surgery, such as endophthalmitis, which could result in an unfavorable visual outcome [3]. Furthermore, as the most frequent complication following cataract surgery, increased corneal edema has been witnessed after uneventful phacoemulsification, with an incidence of $14.3 \%$ [4].

\author{
Corresponding author: \\ Hongyan Ge \\ Department of Eye \\ Eye Hospital \\ First Affiliated Hospital \\ Harbin Medical University \\ 23 Youzheng St \\ 150001 Harbin, China \\ Phone: +860451 85553857 \\ Fax: +86045153650320 \\ E-mail: gehyansh@hotmail. \\ com
}


In our population, the older patients who have poor vision owing to senile cataract are usually suffering from other diseases, mostly diabetes mellitus $[5,6]$. Previous studies have demonstrated that diabetes changed the structure and function as well as the thickness of corneal endothelial cells $[7,8]$. It has also been reported that the presence of macular edema is primarily responsible for the visual outcome after the performance of cataract surgery in diabetic patients [9]. The relationship between corneal endothelial cells and diabetes has been investigated in humans and animals. According to the previous research, the coefficient of variation (CV) of cell area from the normal corneal thickness is significantly associated with the duration of diabetes [10]. Changes in the integrity of corneal endothelial cells and foveal thickness of the macula have been observed after phacoemulsification in diabetic patients and age-matched controls.

The purpose of this study was to evaluate the natural course of changes in corneal endothelial cells and foveal thickness in diabetic and nondiabetic cataract patients during the postoperative period.

\section{Material and methods}

\section{Patients}

The study recruited consecutive patients with cataract scheduled for routine phacoemulsification and intraocular lens (IOL) implantation in our hospital between January, 2008 and October, 2008. Patients who only underwent the ophthalmic surgery were included. Exclusion criteria included any ocular diseases that might influence the monitoring of corneal endothelial cells such as pterygium, high myopia, endothelial dystrophies, keratitis or keratoconus, and any ocular diseases that might influence macular thickness such as uveitis, age-related macular degeneration, intraoperative complications (such as ruptured membranes of posterior lens capsule, dislocation of the lens nucleus into the vitreous space, or bleeding of the epichoroidal space), and inferior quality measurements of optical coherence tomography (OCT) due to media opacities. Additionally, any patient with a history of cataract surgery in the fellow eye during the previous 6 months was also excluded.

Patients were divided into two groups according to the diagnosis of diabetes mellitus type II: the diabetic group and the nondiabetic group. Screening was continued until 60 eyes from each group (one eye per patient) were included for the study. Demographic data such as age and gender were collected. The severity of diabetes was assessed for the diabetic group by measuring the levels of glycated hemoglobin $\left(\mathrm{HbA}_{1 \mathrm{c}}\right)$ and plasma glucose. The study was approved by the Regional Ethics Committee of our hospital, and written informed consent was obtained from all patients prior to their inclusion in the study.

\section{Main outcome measures}

All eyes received a complete ophthalmologic examination on preoperative day 1 (baseline), including best corrected visual acuity (BCVA), Amsler chart, slit-lamp examination, intraocular pressure (IOP) measurement, and dilated fundus examination. The BCVA was recorded on a Snellen chart at each visit.

Corneal endothelial injury associated with phacoemulsification was assessed in terms of cell density and morphology. Photographs of the central corneal endothelium were taken by an automatic specular microscope (Noncon Robo-CA Konan SP-9000p Tokyo, Japan) on a minimum of 40 endothelial cells to evaluate the endothelial cell density (ECD), as well as other parameters including CV in endothelial cell size, percentage of hexagonal cells (PHC) and central corneal thickness (CCT) preoperatively and at day 3, 1 week, and 1,3 , and 6 months postoperatively.

Corneal integrity was also examined using the automatic specular microscope (Noncon Robo-CA Konan SP-9000p Tokyo, Japan), and the central foveal thickness (CFT) was measured and analyzed by Stratus OCT (OCT3, software version 4.0.1; Carl Zeiss Meditec, Dublin, California, USA) as previously described by Hayashi et al. in 2009 [11]. The measurements of corneal integrity and CFT procedures were performed by the same experienced operator immediately before the operation. Three readings were taken for both corneal integrity and $\mathrm{OCT}$, and the average of the three readings was used each time.

Fundus examination was performed at postoperative day 3, 1 week, and 1, 3, and 6 months with full pupil dilation. All patients were examined with fluorescein fundus angiography (FFA) preoperatively and at 12 months postoperatively to confirm the presence of proliferative changes. Macular edema was determined by a physician, also a surgeon, according to four grades: absent (0), focal (1), diffuse (2) and cystoid (3) macular edema [12].

\section{Surgical procedures}

During the cataract surgery, all patients received a hydrophobic acrylic IOL implantation (MA60AC or MA50BM; Alcon Laboratories, Fort Worth, TX, USA).

All the procedures were performed by the same surgeon team under sterile conditions. Tropica- 
mide $(0.5 \%)$ was given for mydriasis 30 min before surgery. Following topical anesthesia (Alcaine), a $3.2-\mathrm{mm}$ clear corneal incision was made at the temporal side using a diamond blade, and the anterior chamber was filled with $2 \%$ methylcellulose (Vistagel, Croma Pharma, Austria). A continuous curvilinear capsulorhexis (approximately $5.0 \mathrm{~mm}$ in diameter) was performed with a bent needle under a viscoelastic material. After hydrodissection, phacoemulsification of the nucleus and cortical aspiration was carried out using a keratome. Then the lens capsule was inflated with $1 \%$ sodium hyaluronate (Healon; Advanced Medical Optics, Santa Ana, CA, USA), followed by the accurate implantation of acrylic IOL into the capsular bag. Finally, all viscoelastic materials were carefully removed. After the surgery, a non-steroid antiinflammatory drug and ophthalmic corticosteroids (Pranopulin Ophthalmic Solution and Loteprednol Etabonate Ophthalmic Suspension) were given for 4 weeks: 4 times per day for the first week, 3 times per day for the second week, twice per day for the third week, and once per day for the last week.

\section{Follow-up}

All patients were followed up at day 3, 1 week, 1 month, 3 months and 6 months postoperatively. During their visits, all patients received a complete ophthalmic examination as they did at preoperative day 1. All baseline and postoperative evaluations were performed by an ophthalmologist (W.L.), who was blinded to the presence of diabetes in patients.

\section{Statistical analysis}

All analyses were performed using SPSS 16.0 (SPSS, Inc., Chicago, IL, USA). The quantitative data are shown as mean \pm standard deviation (SD), and qualitative data are presented as percentages. Comparisons between the diabetic and nondiabetic groups were analyzed by the two-way ANOVA for quantitative data and the $\chi^{2}$ test for qualitative data. Differences were considered statistically significant at $p<0.05$.

\section{Results}

All the patients completed the 6 months follow-up and were all included in the analyses. The demographic and clinical characteristics of these patients are shown in Table I. The diabetic group had a mean duration of diabetes of 4.4 years, and none of the diabetic patients had any severe diabetic complications previously, such as diabetic nephropathy, diabetic retinopathy, diabetic neuropathy, vasculopathy of lower extremities, cardiovascular disease or diabetic foot. They have kept the diabetes under good control according to the criteria of China guideline for type II diabetes (2013 Edition, Table II) [13] by following a healthy diet, exercising regularly and taking oral hypoglycemic agents or insulin treatment. There were no significant differences between diabetic and nondiabetic groups in terms of age, gender, phacoemulsification time, intraocular pressure, nucleus grade, or volume of irrigating solution employed ( $p>0.05$, Table I). No events occurred during all surgical procedures, and no diabetic patients developed proliferative diabetic retinopathy.

Table I. Demographic and intraoperative data of patients with cataract

\begin{tabular}{|c|c|c|}
\hline Parameter & Nondiabetic group & Diabetic group \\
\hline Age [years] & $63.6 \pm 2.4$ & $62.8 \pm 2.2$ \\
\hline \multicolumn{3}{|l|}{ Gender, $n(\%)$ : } \\
\hline Male & $28(46.7)$ & $26(43.3)$ \\
\hline Female & $32(53.3)$ & $34(56.7)$ \\
\hline Duration of diabetes [years] & - & $4.4 \pm 1.5$ \\
\hline Phacoemulsification time [min] & $30.8 \pm 4.2$ & $31.8 \pm 4.0$ \\
\hline Volume of irrigating solution employed [ml] & $122.3 \pm 8.3$ & $126.7 \pm 7.9$ \\
\hline Intraocular pressure at baseline [mm Hg] & $16.9 \pm 2.5$ & $17.6 \pm 2.3$ \\
\hline \multicolumn{3}{|l|}{ Cataract nucleus opalescence (score), $n$ : } \\
\hline 1 & 14 & 12 \\
\hline 2 & 21 & 22 \\
\hline 3 & 22 & 23 \\
\hline 4 & 3 & 3 \\
\hline
\end{tabular}

Data are presented as mean $\pm S D$ or percentage. 
Table III presents the comparison of mean ECD, $\mathrm{PHC}, \mathrm{CV}, \mathrm{CCT}$ and CFT, and all parameters showed no difference between the nondiabetic and diabetic groups before surgery $(p>0.05)$. During the 6 months of follow-up, both groups had decreased ECD and PHC and increased CV after surgery (Figure $1 ; F_{E C D}=151.73, F_{P H C}=718.42$ and $F_{C V}$ $=126.42$, respectively; all $\left.p_{\text {time }}<0.05\right)$, and the diabetic group had more changes in ECD, PHC, and $C V$ than the nondiabetic group at each time point postoperatively $\left(F_{E C D}=56.03, F_{P H C}=382.43\right.$ and $F_{C V}=59.25$, respectively; all $\left.p_{\text {group }}<0.05\right)$. The CCT and CFT fluctuated in both groups during the follow-up (Figure 2; $F_{C C T}=87.91$ and $F_{C F T}=441.68$, respectively; both $p_{\text {time }}<0.05$ ), and the diabetic group had individual peaks of CCT and CFT both at postoperative 1 week. Additionally, the diabetic group had a thicker central cornea during the postoperative 6 months and thicker central fovea within postoperative 1 month than the nondiabetic group $\left(F_{C C T}=69.33\right.$ and $F_{C F T}=393.05$, respectively; all $p_{\text {group }}<0.05$; Table III). Finally, the central fovea in both groups gradually recovered to normal thickness from postoperative 3 months. Furthermore, significant interactions of time and group were observed for ECD, CV, PHC, CCT and CFT $\left(F_{E C D}=133.04, F_{P H C}=545.46, F_{C V}=76.96, F_{C C T}=52.09\right.$ and $F_{C F T}=423.62$, respectively; all $\left.p_{\text {group } \times \text { time }}<0.05\right)$.

\section{Discussion}

This study evaluated the natural course of changes in corneal endothelial cells and foveal thickness in diabetic and nondiabetic patients after cataract surgery. The results showed that corneal integrity significantly changed in both nondiabetic and diabetic groups after surgery and that ECD, PHC, CV, CCT and CFT were obviously different in the diabetic and nondiabetic groups during the postoperative observation period.

The corneal endothelium is the innermost layer of the cornea that comprises a monolayer of flat, nonmitotic polygonal cells (mostly hexagonal cells) and is essential for maintenance of normal corneal hydration, thickness and transparency by its barrier and pump functions $[14,15]$. Corneal endothelial cells are embryologically derived from the neural crest [16]. They can secrete mucopolysaccharide stromal collagen fibers at an osmotic pressure of $60 \mathrm{~mm} \mathrm{Hg}$ to maintain the relatively dehydrated state; hence, a finite number of endothelial cells is the key to the corneal epithelium and its normal transit function [17, 18]. However, they can be damaged by injury and pathologies such as intraocular surgery and Fuchs dystrophy, but are not regenerated due to their very limited proliferative capacity [19]. Many studies have confirmed that endothelial cells cannot regenerate but migrate and expand through the elastic layer around the cell to compensate for
Table II. Goal of comprehensive control of type II diabetes in China

\begin{tabular}{|c|c|}
\hline Parameter & Target value \\
\hline \multicolumn{2}{|l|}{ Blood glucose [mmol/l]; } \\
\hline Fasting & $4.4-7.0$ \\
\hline Non-fasting & $<10.0$ \\
\hline Glycosylated hemoglobin $\left(\mathrm{HbA}_{1 \mathrm{c}}, \%\right)$ & $<7.0$ \\
\hline Blood pressure [mm Hg] & $<140 / 80$ \\
\hline Total cholesterol (TC) [mmol/l] & $<4.5$ \\
\hline Triglyceride (TG) [mmol/l) & $<1.5$ \\
\hline Body mass index $\left[\mathrm{kg} / \mathrm{m}^{2}\right]$ & $<24.0$ \\
\hline \multicolumn{2}{|l|}{ Urinary albumin/creatinine [mg/day]: } \\
\hline Male & $<2.5(22.0)$ \\
\hline Female & $<3.5(31.0)$ \\
\hline $\begin{array}{l}\text { Urinary albumin excretion rate (AER) } \\
{[\mu \mathrm{g} / \mathrm{min}(\mathrm{mg} / \text { day })]}\end{array}$ & $<20.0(30.0)$ \\
\hline
\end{tabular}

the loss of endothelial cells [20]. Over the course of this expansion, irreversible edema and opacity might develop in the cornea, which is a serious eye condition. The loss of corneal endothelial cells is influenced by various factors, such as surgeon's experience, hardness of cataract, type of corneal incision, age and preoperative visual acuity [21]. Therefore, corneal ECD is often detected in intraocular surgery to guide the choice of surgical approach, especially before and after surgery for cataracts, glaucoma and other significant disorders.

Also, the cell area was an important indicator for the evaluations of endothelial cell function and corneal functional recovery. A high CV of the endothelial deformation index indicated that cells were of varying size and that there were large size differences between them. Therefore, the increase in $\mathrm{CV}$ of the corneal endothelial cell area was a sensitive indicator of corneal endothelial cell rearrangement [22], whereas the corneal thickness was an important indicator of endothelial function [23]. As shown in this study, the diabetic group had more changes in ECD, PHC and CV after surgery compared with the nondiabetic group, suggesting that endothelial cells have been migrating and expanding after their loss, and this rearrangement could be affected by diabetes.

In this study, diabetic patients have poor corneal endothelial function as evidenced by the higher CCT during the 6 months postoperatively compared with the nondiabetic group. In general, the cornea depends on endothelial cell sodium-potassium ATPase pumps to maintain the balance of the matrix of ions and water. The cornea is always 
Zhuo Chen, Fanqian Song, Liyao Sun, Chuchu Zhao, Ningning Gao, Ping Liu, Hongyan Ge

Table III. Changes in corneal endothelial morphology and thickness after cataract surgery

\begin{tabular}{|c|c|c|c|}
\hline Parameter & Nondiabetic group & Diabetic group & $P$-value \\
\hline \multicolumn{4}{|l|}{ Endothelial cell density [cells $/ \mathrm{m}^{2}$ ]: } \\
\hline Preoperative day 1 (baseline) & $2742.14 \pm 107.54$ & $2743.56 \pm 109.23$ & 0.943 \\
\hline Postoperative day 3 & $2740.92 \pm 107.32$ & $2628.07 \pm 124.02$ & $<0.001$ \\
\hline Postoperative 1 week & $2738.36 \pm 108.23$ & $2575.81 \pm 129.97$ & $<0.001$ \\
\hline Postoperative 1 month & $2736.85 \pm 108.89$ & $2524.49 \pm 132.23$ & $<0.001$ \\
\hline Postoperative 3 months & $2733.97 \pm 109.12$ & $2505.17 \pm 146.90$ & $<0.001$ \\
\hline Postoperative 6 months & $2733.73 \pm 108.98$ & $2503.64 \pm 104.61$ & $<0.001$ \\
\hline \multicolumn{4}{|l|}{ Percentage of hexagonal cells (\%): } \\
\hline Preoperative day 1 (baseline) & $56.95 \pm 1.61$ & $57.02 \pm 1.57$ & 0.808 \\
\hline Postoperative day 3 & $56.71 \pm 1.62$ & $53.63 \pm 1.87$ & $<0.001$ \\
\hline Postoperative 1 week & $56.55 \pm 1.63$ & $51.11 \pm 1.49$ & $<0.001$ \\
\hline Postoperative 1 month & $56.46 \pm 1.65$ & $49.42 \pm 1.00$ & $<0.001$ \\
\hline Postoperative 3 months & $56.42 \pm 1.64$ & $49.30 \pm 1.03$ & $<0.001$ \\
\hline Postoperative 6 months & $56.37 \pm 1.66$ & $49.26 \pm 1.05$ & $<0.001$ \\
\hline \multicolumn{4}{|c|}{ Coefficient of variation in endothelial cell size (\%): } \\
\hline Preoperative day 1 (baseline) & $43.29 \pm 1.85$ & $43.52 \pm 2.00$ & 0.533 \\
\hline Postoperative day 3 & $43.41 \pm 1.80$ & $45.83 \pm 2.89$ & $<0.001$ \\
\hline Postoperative 1 week & $43.63 \pm 1.84$ & $46.85 \pm 3.11$ & $<0.001$ \\
\hline Postoperative 1 month & $43.80 \pm 1.88$ & $48.60 \pm 3.84$ & $<0.001$ \\
\hline Postoperative 3 months & $43.95 \pm 1.92$ & $49.00 \pm 3.80$ & $<0.001$ \\
\hline Postoperative 6 months & $43.97 \pm 1.89$ & $49.11 \pm 3.83$ & $<0.001$ \\
\hline \multicolumn{4}{|l|}{ Central corneal thickness $[\mu \mathrm{m}]$ : } \\
\hline Preoperative day 1 (baseline) & $511.58 \pm 13.59$ & $513.36 \pm 16.16$ & 0.519 \\
\hline Postoperative day 3 & $512.80 \pm 13.86$ & $540.07 \pm 20.70$ & $<0.001$ \\
\hline Postoperative 1 week & $512.69 \pm 13.46$ & $547.95 \pm 27.26$ & $<0.001$ \\
\hline Postoperative 1 month & $506.49 \pm 9.05$ & $540.27 \pm 20.79$ & $<0.001$ \\
\hline Postoperative 3 months & $506.46 \pm 8.53$ & $511.90 \pm 15.71$ & 0.022 \\
\hline Postoperative 6 months & $502.37 \pm 4.28$ & $513.44 \pm 16.10$ & $<0.001$ \\
\hline \multicolumn{4}{|l|}{ Central foveal thickness $[\mu \mathrm{m}]$ : } \\
\hline Preoperative day 1 (baseline) & $195.03 \pm 2.91$ & $194.97 \pm 2.87$ & 0.908 \\
\hline Postoperative day 3 & $195.53 \pm 2.75$ & $208.86 \pm 5.30$ & $<0.001$ \\
\hline Postoperative 1 week & $195.53 \pm 2.75$ & $232.98 \pm 11.79$ & $<0.001$ \\
\hline Postoperative 1 month & $195.89 \pm 2.70$ & $217.25 \pm 7.45$ & $<0.001$ \\
\hline Postoperative 3 months & $195.84 \pm 2.64$ & $195.74 \pm 2.64$ & 0.850 \\
\hline Postoperative 6 months & $195.02 \pm 2.90$ & $195.00 \pm 2.87$ & 0.959 \\
\hline
\end{tabular}

Data are presented as mean $\pm S D$. Differences were considered statistically significant at $p<0.05$. 
A

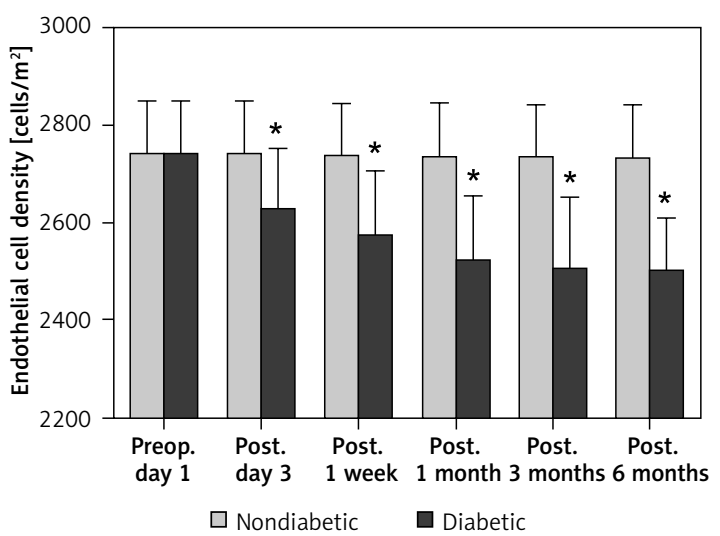

C

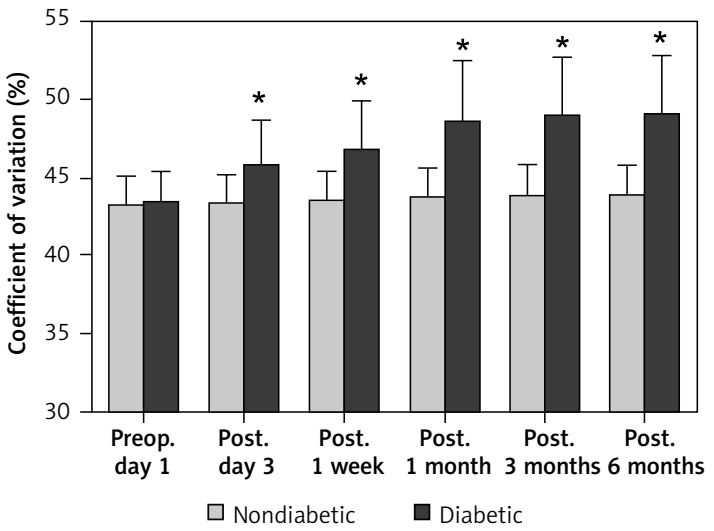

A

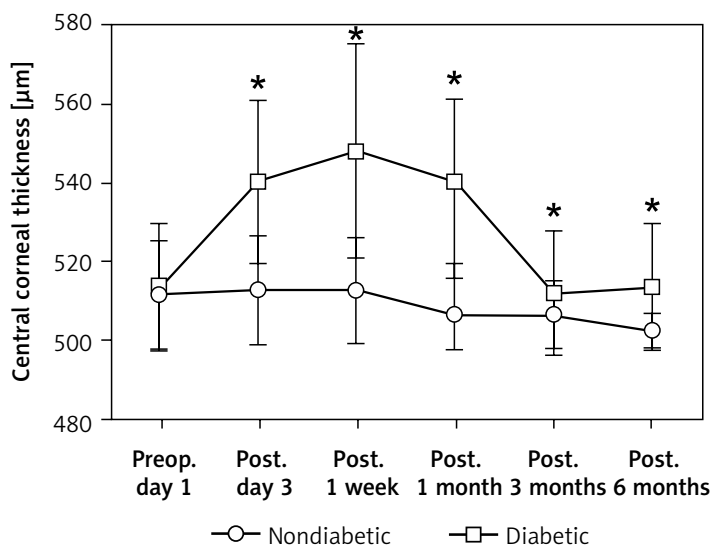

B

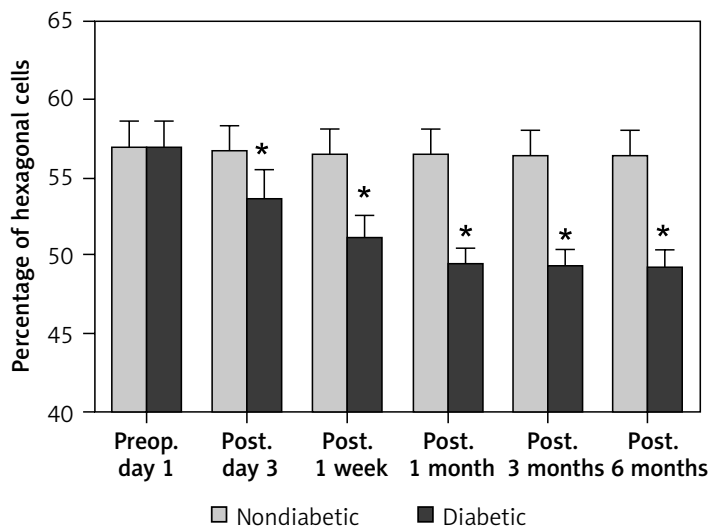

Figure 1. Comparisons of endothelial cell density (A), percentage of hexagonal cells (B), and coefficient of variation $(\mathbf{C})$ between the diabetic and nondiabetic groups at preoperative day 1 and postoperative day 3, 1 week, 1 month, 3 months, and 6 months. Data are shown as mean \pm standard error (SE). *Diabetic group differed from nondiabetic group postoperatively at the time point $\left(p_{\text {group }}<0.05\right)$

\section{B}

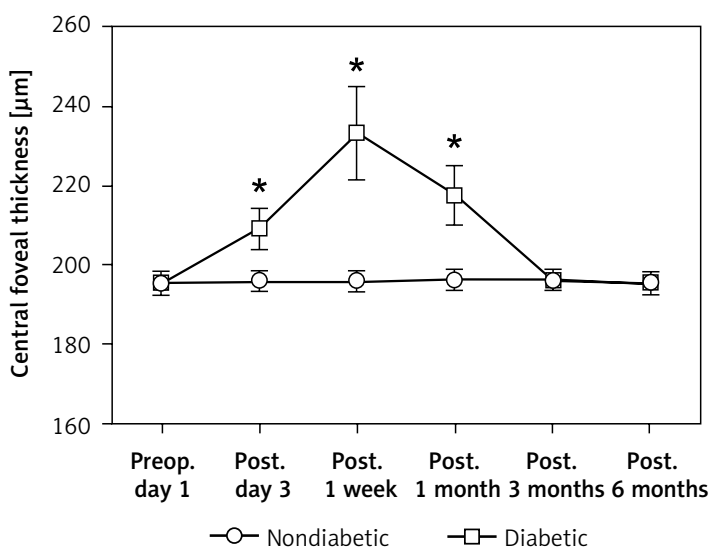

Figure 2. Comparison of central corneal thickness (A) and central foveal thickness (B) between the diabetic and nondiabetic groups at preoperative day 1 and postoperative day 3, 1 week, 1 month, 3 months, and 6 months. Data are shown as mean \pm standard error (SE). *Diabetic group differed from nondiabetic group postoperatively at the time point $\left(p_{\text {group }}<0.05\right)$

in a relatively dehydrated state, which maintains its transparency. $\mathrm{Na}^{+} / \mathrm{K}^{+}$ATPase activity leading to cell pump dysfunction is an important mechanism of diabetic corneal edema. Similarly, Herse found that $69 \%$ to $76 \%$ of endothelial cells in diabetic animal models had reduced $\mathrm{Na}^{+} / \mathrm{K}^{+}$ATP enzyme activity [24], which could cause diabetic corneal thickening, not because of the increase in matrix components but due to the increase in water penetration. Moreover, postoperative corneal edema has been associated with many specific factors, for example, Tsaousis et al. found that ultrasound energy was a defining factor for the development of corneal edema apart from diabetes mellitus type II, although the consumed ultrasound energy did not define the final clinical outcome [4]. 
In this study, the increase in CFT within 1 month postoperatively was greater in patients with diabetes than those without diabetes, indicating that diabetic macular edema occurred after cataract surgery and was worse in eyes with diabetes. Then, CFT in both groups gradually recovered to normal at postoperative 3 months. This could be demonstrated by previous research in which diabetic macular edema developed in approximately $20-40 \%$ of patients receiving cataract surgery, but the macular edema resolved spontaneously in a considerable percentage of these patients $[12,25]$. These findings indicated the presence of macular thickening usually following cataract surgery and its regression with time in most diabetic patients. However, diabetic maculopathy actually progresses in some diabetic patients. Both types of macular changes are more prominent in patients with diabetes than those without.

Patients with diabetes who have a normal corneal epithelium, epithelial basement membrane and basement membrane complex may experience morphological changes in the basal layer and endothelium because their own balance of these structures is changed. These changes cause the primary or secondary (surgery) corneal changes, which is why it is more difficult for patients with diabetes to withstand the trauma of eye surgery.

There were several limitations in this study. For example, $\mathrm{HbA}_{1 \mathrm{c}}$ and the mean value of blood glucose levels were not analyzed although these diabetic patients have kept the diabetes under good control. Also, perioperative and postoperative glucose variability were not detected, and further study is needed to investigate whether glucose variability is an effective factor for postoperative changes in diabetic patients. Notwithstanding its limitations, this study demonstrated that diabetic patients were prone to have more changes in the corneal endothelial morphology and thickness, and an increased foveal thickness after phacoemulsification with implantation of a foldable IOL compared to nondiabetic patients. Therefore, one should be alert when performing phacoemulsification surgery in diabetic patients with cataract to prevent the direct effects of the acoustic vibration, heat, and objects.

\section{Acknowledgments} thors.

Zhuo Chen and Fanqian Song are co-first au-

This research was supported by the Special Fund for the Doctoral Program of Higher Education (New Teacher category: 20132307120035), and the Natural Science Foundation of China (Project No. 81300728).

\section{Conflict of interest}

The authors declare no conflict of interest.

\section{References}

1. Organization WH. Visual impairment and blindness. Fact Sheet 2011; 2009-10.

2. Chung YW, Oh TH, Chung SK. The effect of topical cyclosporine $0.05 \%$ on dry eye after cataract surgery. Korean J Ophthalmol 2013; 27: 167-71.

3. Yu-Wai-Man P, Morgan SJ, Hildreth AJ, Steel DH, Allen D. Efficacy of intracameral and subconjunctival cefuroxime in preventing endophthalmitis after cataract surgery. J Cataract Refract Surg 2008; 34: 447-51.

4. Tsaousis KT, Panagiotou DZ, Kostopoulou E, Vlatsios V, Stampouli D. Corneal oedema after phacoemulsification in the early postoperative period: a qualitative comparative case-control study between diabetics and non-diabetics. Ann Med Surg 2016; 5: 67-71.

5. Olafsdottir E, Andersson DK, Stefánsson E. The prevalence of cataract in a population with and without type 2 diabetes mellitus. Acta Ophthalmol 2012; 90: 334-40.

6. Prokofyeva E, Wegener A, Zrenner E. Cataract prevalence and prevention in Europe: a literature review. Acta Ophthalmol 2013; 91: 395-405.

7. Hugod M, Storr-Paulsen A, Norregaard JC, Nicolini J, Larsen AB, Thulesen J. Corneal endothelial cell changes associated with cataract surgery in patients with type 2 diabetes mellitus. Cornea 2011; 30: 749-53.

8. Storr-Paulsen A, Singh A, Jeppesen H, Norregaard JC, Thulesen J. Corneal endothelial morphology and central thickness in patients with type II diabetes mellitus. Acta Ophthalmol 2014; 92: 158-60.

9. Takamura Y, Kubo E, Akagi Y. Analysis of the effect of intravitreal bevacizumab injection on diabetic macular edema after cataract surgery. Ophthalmology 2009; 116: 1151-7.

10. Schultz RO, Matsuda M, Yee RW, Edelhauser HF, Schultz KJ. Corneal endothelial changes in type I and type II diabetes mellitus. Am J Ophthalmol 1984; 98: 401-10.

11. Hayashi K, Igarashi C, Hirata A, Hayashi H. Changes in diabetic macular oedema after phacoemulsification surgery. Eye 2009; 23: 389-96.

12. Funatsu $H$, Yamashita $H$, Noma $H$, Shimizu E, Mimura $T$, Hori S. Prediction of macular edema exacerbation after phacoemulsification in patients with nonproliferative diabetic retinopathy. J Cataract Refract Surg 2002; 28: 1355-63.

13. Chinese Diabetes Society. China guideline for type 2 diabetes (2013 Edition). Chin J Diabetes 2014; 22: 2-42.

14. Dhasmana R, Singh I, Nagpal RC. Corneal changes in diabetic patients after manual small incision cataract surgery. J Clin Diagn Res 2014; 8: VC03-6.

15. Bucht C, Söderberg P, Manneberg G. Simulation of specular microscopy images of corneal endothelium, a tool for control of measurement errors. Acta Ophthal 2011; 89: e242-50.

16. Chou ML, Burnouf T, Wang TJ. Ex vivo expansion of bovine corneal endothelial cells in xeno-free medium supplemented with platelet releasate. PloS One 2014; 9: e99145.

17. Sudhir RR, Raman R, Sharma T. Changes in the corneal endothelial cell density and morphology in patients with type 2 diabetes mellitus: a population-based study, Sankara Nethralaya Diabetic Retinopathy and Molecu- 
lar Genetics Study (SN-DREAMS, Report 23). Cornea 2012; 31: 1119-22.

18. Goktas A, Gumus K, Mirza GE, Crockett C, Karakucuk S, Cavanagh HD. Corneal endothelial characteristics and central corneal thickness in a population of Turkish cataract patients. Eye Contact Lens 2012; 38: 142-5.

19. Joyce NC. Proliferative capacity of the corneal endothelium. Prog Retin Eye Res 2003; 22: 359-89.

20. Kim DH, Wee WR, Hyon JY. The pattern of early corneal endothelial cell recovery following cataract surgery: cellular migration or enlargement? Graef Arch Clin Exp 2015; 253: 2211-6.

21. Orski M, Synder A, Pałenga-Pydyn D, Omulecki W, Wilczyński $M$. The effect of the selected factors on corneal endothelial cell loss following phacoemulsification. Klin Oczna 2013; 116: 94-9.

22. Waring GO, Bourne WM, Edelhauser HF, Kenyon KR. The corneal endothelium: normal and pathologic structure and function. Ophthalmology 1982; 89: 531-90.

23. Patel SV, Hodge DO, Bourne WM. Corneal endothelium and postoperative outcomes 15 years after penetrating keratoplasty. Am J Ophthalmol 2005; 139: 311-9.

24. Herse PR. Corneal hydration control in normal and alloxan-induced diabetic rabbits. Invest Ophthalmol Vis Sci 1990; 31: 2205-13.

25. Squirrell D, Bhola R, Bush J, Winder S, Talbot J. A prospective, case controlled study of the natural history of diabetic retinopathy and maculopathy after uncomplicated phacoemulsification cataract surgery in patients with type 2 diabetes. Br J Ophthalmol 2002; 86: 565-71. 